SAT0457

SJÖGREN BIG DATA PROJECT, THE FIRST EXAMPLE OF DATA SHARING IN AUTOIMMUNE DISEASES: ANALYSIS OF 10475 WORLDWIDE PATIENTS

S. Retamozo ${ }^{1,2,3}$, N. Acar-Denizli ${ }^{4}$, W. Fai Ng ${ }^{5}$, M. Zeher ${ }^{6}$, A. Rasmussen ${ }^{7}$, T. Mandl ${ }^{8}$, R. Seror ${ }^{9}$, X. Li $^{10}$, C. Baldini ${ }^{11}$, J.-E. Gottenberg ${ }^{12}$, D. Danda ${ }^{13}$ L. Quartuccio ${ }^{14}$, A. Minniti ${ }^{15}$, G. Hernandez-Molina ${ }^{16}$, U. Kalyoncu ${ }^{17}$, A.A. Kruize ${ }^{18}$, S.-K. Kwok ${ }^{19}$, M. Wahren-Herlenius ${ }^{20}$, S. Praprotnik ${ }^{21}$, D. Sene ${ }^{22}$, E. Bartoloni ${ }^{23}$, R. Solans ${ }^{24}$, M. Rischmueller ${ }^{25}$, Y. Suzuki ${ }^{26}$, D. Isenberg ${ }^{27}$, V. Valim ${ }^{28}$ P. Wiland ${ }^{29}$, G. Nordmark ${ }^{30}$, G. Fraile $^{31}$, H. Bootsma ${ }^{32}$, T. Nakamura ${ }^{33}$, R. Giacomelli ${ }^{34}$, V. Devauchelle-Pensec ${ }^{35}$, B. Hofauer ${ }^{36}$, M. Bombardieri $^{37}$, V. Fernandes Moça Trevisani ${ }^{38}$, D. Hammenfors ${ }^{39}$, S.G. Pasoto ${ }^{40}$, T.A. Gheita ${ }^{41}$ F. Atzeni ${ }^{42}$, J. Morel ${ }^{43}$, C. Vollenveider ${ }^{44}$, P. Brito-Zerón ${ }^{1,45}$, M. Ramos-Casals' ${ }^{1}$. ${ }^{1} \mathrm{H}$. Clinic, IDIBAPS, Barcelona, Spain; ${ }^{2}$ Hosp Univ Córdoba, IUCBC; ${ }^{3} I N I C S A$, UNC, CONICET, Cordoba, Argentina; ${ }^{4}$ Mimar Sinan Univ, Istanbul, Turkey; ${ }^{5}$ Newcastle Univ, NHS Foundation Trust, Newcastle, UK; ${ }^{6}$ Debrecen Univ, Debrecen, Hungary, ${ }^{7}$ Oklahoma Medical Research Foundation, Oklahoma, USA; ${ }^{8}$ Malmö Hosp, Lund Univ, Lund, Sweden; ${ }^{9}$ Université Paris Sud, INSERM, Paris, France; ${ }^{10}$ Anhui Provincial Hosp, Hefei, China; ${ }^{11}$ Pisa Univ, Pisa, Italy,

${ }^{12}$ Strasbourg Univ, CNRS, Strasbourg, France; ${ }^{13}$ Christian Medical College and Hosp, Vellore, India; ${ }^{14}$ Hosp "Santa Maria della Misericordia", Udine; ${ }^{15}$ Sapienza Univ, Rome, Italy; ${ }^{16}$ INCMNSS, Mexico, Mexico; ${ }^{17}$ Hacettepe Univ, Ankara, Turkey, ${ }^{18}$ Univ Medical Center, Utrecht, Netherlands; ${ }^{19}$ Catholic Univ of Korea, Seoul, Korea, Republic Of, ${ }^{20}$ Karolinska Institute, Stockholm, Sweden; ${ }^{21}$ Univ Medical Centre, Ljubljana, Slovenia; ${ }^{22}$ Univ Paris VII Publique-Hôpitaux de Paris, 2, Paris, France; ${ }^{23}$ Perugia Univ, Perugia, Italy; ${ }^{24} \mathrm{H}$. Vall d'Hebron, Barcelona, Spain; ${ }^{25}$ Western Australia Univ, Crawley, Australia; ${ }^{26}$ Kanazawa Univ Hosp, Ishikawa, Japan; ${ }^{27}$ University College, London, UK; ${ }^{28}$ Federal Univ Espírito Santo, Vitória, Brazil; ${ }^{29}$ Wroclaw Medical Hospital, Wroclaw, Poland; ${ }^{30}$ Uppsala Univ, Uppsala, Sweden; ${ }^{31}$ H. Ramón y Cajal, Madrid, Spain; ${ }^{32}$ Univ Medical Center, Groningen, Netherlands; ${ }^{33}$ Nagasaki University, Nagasaki, Japan; ${ }^{34}$ L'Aquila Univ, L'Aquila, Italy; ${ }^{35}$ Brest Univ Hosp, CERAINO, Brest, France, ${ }^{36}$ Technische Univ, München, Germany; ${ }^{37}$ Queen Mary Univ, London, UK; ${ }^{38}$ Federal University of São Paulo, Sao Paulo, Brazil; ${ }^{39}$ Haukeland Univ Hosp, Bergen, Norway, ${ }^{40}$ Hospital das Clínicas, USP, São Paulo, Brazil; ${ }^{41}$ Cairo University, Cairo, Egypt, ${ }^{42}$ Messina and Milan Univ, Milan, Italy, ${ }^{43} \mathrm{Hosp}$ and Univ of Montpellier, Montpellier, France;

${ }^{44}$ German Hosp, Buenos Aires, Argentina; ${ }^{45} \mathrm{H}$. CIMA-Sanitas, Barcelona, Spain

Objectives: To take a "high-definition" picture of the main features of primary Sjögren syndrome (SjS) following a worldwide data-sharing cooperative merging of international clinical SjS databases.

Methods: The Big Data Sjögren Project Consortium is an international, multicentre registry created in 2014 including leading clinical centres in SjS of the 5 continents that shared a harmonised data architecture and conducted cooperative online efforts to refine collected data of primary SjS patients fulfilling the 2002 classification criteria.

Results: By January 2018, the participant centres had included 10475 patients from 22 countries, including 7637 (73\%) patients from Europe, 1420 (14\%) from America, $1186(11 \%)$ from Asia, $167(1.4 \%)$ from Australia and $65(0.6 \%)$ from Africa. The cohort included 9781 (93\%) women and 694 (7\%) men, with a mean age at diagnosis of primary SjS of 53 years. The frequencies of fulfilment of the 2002 criteria were $92 \%$ for dry eye, $94 \%$ for dry mouth, $83 \%$ for abnormal ocular tests, $82 \%$ for positive minor salivary gland biopsy, $78 \%$ for abnormal oral diagnostic tests and $76 \%$ for positive anti-Ro/La antibodies. The frequency of positive immunological markers at diagnosis was $79 \%$ for ANA, $73 \%$ for anti-Ro, $49 \%$ for RF, $45 \%$ for anti-La, $13 \%$ for low C3 levels, $14 \%$ for low C4 levels and $7 \%$ for cryoglobulins.

Conclusions: International data sharing-based projects merging disperse clinical registries may be essential tools to increase current knowledge and to improve patient care in specific systemic autoimmune diseases.

Disclosure of Interest: None declared

DOI: 10.1136/annrheumdis-2018-eular.6018

\section{SAT0458 URINE SOLUBLE UROKINASE PLASMINOGEN ACTIVATOR RECEPTOR AS A POTENTIAL BIOMARKER OF LUPUS NEPHRITIS ACTIVITY}

S. Burcsár ${ }^{1}$, G. Toldi ${ }^{2}$ B. Szalay ${ }^{3}$, B. Vásárhelyi ${ }^{3}$, L. Kovács ${ }^{1}$, A. Balog ${ }^{1}$ ${ }^{1}$ Department of Rheumatology and Immunology, Faculty of Medicine, Albert SzentGyörgyi Health Center, University of Szeged, Szeged, Hungary; ${ }^{2}$ Neonatal Unit, Birmingham Women's and Children's Hospital, Birmingham, UK; ${ }^{3}$ Department of Laboratory Medicine, Semme/weis University, Budapest, Hungary

Background: Over the recent years, soluble urokinase plasminogen activator receptor (suPAR) has been described as a valuable indicator of the activation state of the immune system. Our previous work supported that suPAR might be an objective marker for identifying systemiclupus erythematosus (SLE) patients with active disease. Urokinase plasminogen activator receptor (UPAR) expression is also induced in podocytes during renal diseases such as focal segmental glomerulosclerosis (FSGS). In addition, levels of suPAR were found to be elevated in paediatric and adult patients with FSGS. Current noninvasive laboratory and clinical measures of lupus nephritis (LN) activity are not sensitive or specific enough to reliably measure the course of LN. Improved, noninvasive biomarkers are needed to accurately detect $L N$ activity

Objectives: To evaluate suPAR in urine as a potential biomarker of renal systemspecific disease activity in SLE.

Methods: A prospective follow-up study was designed. 14 SLE patients with newly diagnosed $L N$ and 31 healthy individuals were enrolled in this study. LN was the leading symptom of all SLE patients. Further organ involvements including arthritis, leucopenia, serositis, fever and rash also occurred at the time of LN diagnosis. 12 out of $14 \mathrm{LN}$ were confirmed by renal biopsy. Urine samples were taken before the initiation of $L N$ induction therapy, and monthly thereafter (6 to 11 times).Global and renal disease activity were defined using the SLE Disease Activity Index 2000 (SLEDAI-2K) and the SLEDAI-2K renal domain score. suPAR concentrations in the urine were measured with the suPARnostic Flex ELISA assay. Nonparametric testing was used for statistical analysis, including the Kruskal-Wallis test and the Mann-Whitney U test.

Results: All urine suPAR levels were elevated in SLE patients with active LN compared with patients with resolved $L N$ and healthy controls ( 21.75 vs 4.93 and $5.09 \mathrm{ng} / \mathrm{mL}$, respectively, $\mathrm{p}<0.0001)$. suPAR levels were higher measured before than after the initiation of LN induction therapy in patients before resolution of active LN (29.46 vs $21.07 \mathrm{ng} / \mathrm{mL}, \mathrm{p}=0.0122$ ). Prospective follow-up measurements also suggested that urine suPAR levels raised again in patients with a relapse of $L N$ according to SLEDAI-2K renal domain score $(n=3 ; 5.45$ vs $15.19 \mathrm{ng} / \mathrm{mL}, \mathrm{p}=0.0127$ ).

Conclusions: Urine suPAR is a promising $L N$ activity biomarker, given its isolated elevation in urine in active $L N$ and pronounced decrease with $L N$ improvement. It may potentially become a more convenient alternative to renal biopsy, the current gold standard of $\mathrm{LN}$ activity monitoring.

Acknowledgements: Attila Balog was supported by the János Bolyai Scholarship. This work was supported by the GINOP-2.3.2-15-2016-00 030 research found.

Disclosure of Interest: None declared

DOI: 10.1136/annrheumdis-2018-eular.2283

\section{SAT0459 \\ OCCURRENCE OF LYMPHOMA IN SYSTEMIC LUPUS ERYTHEMATOSUS: A CASE-SERIES OF 38 PATIENTS}

T. Huscenot ${ }^{1}$, A. Mathian ${ }^{1}$, M. Martin ${ }^{2}$, C. Deligny ${ }^{3}$, J.-S. Allain ${ }^{4}$, M. Pha ${ }^{1}$, N. Costedoat-Chalumeau ${ }^{5}$, V. Le Guern ${ }^{5}$, G. Pugnet ${ }^{6}$, F. Cohen-Aubart ${ }^{1}$,

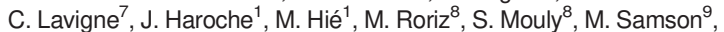
H. Devilliers ${ }^{9}$, B. Bonnotte ${ }^{9}$, M. Versini ${ }^{10}$, P.-Y. Jeandel ${ }^{10}$, A.-S. Korganow ${ }^{2}$ T. Martin ${ }^{2}$, V. Leblond ${ }^{11}$, F. Charlotte ${ }^{11}$, S. Choquet ${ }^{11}$, Z. Amoura ${ }^{1}{ }^{1}$ Service de Médecine Interne, centre de référence Lupus et Syndrome des Antiphospholipides, Hôpital La Pitié-Salpétrière, Paris; ${ }^{2} \mathrm{CHRU}$ de Strasbourg, Strasbourg; ${ }^{3} \mathrm{CHU}$ de Martinique, Martinique; ${ }^{4} \mathrm{CHU}$ de Rennes, Rennes; ${ }^{5} \mathrm{Hôpital}$ Cochin, Paris: ${ }^{6} \mathrm{CHU}$ Purpan, Toulouse; ${ }^{7} \mathrm{CHU}$ de Angers, Angers; ${ }^{8}$ Groupe Hospitalier LariboisièreFernand Vidal, Paris; ${ }^{9} \mathrm{CHU}$ de Dijon, Dijon; ${ }^{10} \mathrm{CHU}$ de Nice, Nice; ${ }^{11}$ Hôpital la PitiéSalpétrière, Paris, France

Background: Patients with systemic lupus erythematosus (SLE) are at higher risk than the general population of developing lymphoma. Relatively little is known about the risk factors, the treatment and the outcome of lymphoma in SLE.

Objectives: We aimed to describe a cohort of patients suffering from lymphoma in the setting of SLE and to study the risk factors of developing this complication. Methods: We collected clinical data of SLE patients with confirmed lymphoma in a multicentric and retrospective study. SLE patients were eligible for the study if they fulfilled at least 4 of the 1997 ACR criteria for SLE. Exclusion criteria were HIV or $\mathrm{C}$ hepatitis infection.

Results: We included 38 patients ( 34 women and 4 men) coming from 10 different French University Hospitals. The lymphoma occurred after the diagnosis of SLE for 35 patients, with a median (range) time of 8.8 years (0-39). In their past or present medical history, $11(29 \%)$ had a haematological involvement (5 immune thrombocytopenias, 4 autoimmune hemolytic anemias and 2 patients with both). Nine patients (24\%) had associated Sjögren syndrome. 22 patients (58\%) had a polyclonal hypergammaglobulinemia. Before the occurrence of the lymphoma, 18 patients $(47 \%)$ had received an immunosuppressant during a median (range) period of 67 months. ${ }^{6-195} 17$ patients had an indolent $B$ cell lymphoma (IBCL), 14 a high-grade $B$ cell lymphoma (11 diffuse large $B$ cell lymphoma [DLBCL]; 2 a primary DLBCL of the central nervous system; and 1 an iatrogenic immunodeficiency-associated lymphoproliferative disorder), 5 a Hodgkin's disease (HD), and 2 a T cell lymphoma. The EBER in situ hybridization stain was positive in 7 of the 13 patients assessed $(3 / 3 \mathrm{HD}, 3 / 7 \mathrm{DLBCL}$ and $1 / 2 \mathrm{IL})$ and was not associated with the immunosuppressant prescription. The median (range) of the survey after the lymphoma was 28.5 month (0-235.1). The 14 DLBCL patients except 1 were treated with chemotherapy: 4 died, 9 were in complete remission and 1 was in progression. Regarding the 17 IBCL, 9 patients were treated with chemotherapy, 2 